\title{
Technological Report
}

\section{Properties of Layered Inorganic Ion Exchangers with an Intercalated Antifungal Agent}

\author{
Yasuharu ONO \\ SPECIALTY PRODUCTS GROUP, SPECLALTY CHEMICALS DEPERTMENT, TOAGOSEI CO., LTD., \\ 1-1 FUNAMI-CHO, MINATO-KU, NAGOYA 455-0027, JAPAN
}

(Manuscript submitted February 14, 2003; accepted April 9, 2003)

\begin{abstract}
We made trials on several kinds of ions placed between the layers of synthetic fluoride substituted mica (abbreviated to MICA), with an organic antifungal agent intercalated, to synthesize an antifungal phyllosilicate with high thermal stability and effect. Bonds of an organic antifungal agent and MICA were classified into two types, an intermolecular bond and an ionic bond. The former was the case when ions between layers are $\mathrm{Na}^{+}$and $\mathrm{Ca}^{2+}$, and the latter was the case of $\mathrm{Cu}^{2+}$. The compound with an intermolecular bond showed a tendency to have comparatively high antifungal effect. In the case of the intermolecular bond, when ions between the layers of a compound were $\mathrm{Ca}^{2+}$, thermal stability of the antifungal agent improved, but it was not so when ions were $\mathrm{Na}^{+}$.
\end{abstract}

\section{Introduction}

Organic antifungal agents have high effects, and have broad applications. But as they easily decompose by heat or light (ultraviolet rays), few of them can be knead into plastics. So they often fail to show antifungal effect. On the other hand, layered inorganic ion exchangers, such as montmorillonite, are known to intercalate various substances into their layers.[1,2] If organic antifungal agents are intercalated in their layers, improvement in heat resistance can be expected. We made trials of intercalating organic antifungal agents into layered inorganic ion exchangers to examine physical properties of synthesized compounds. We report here the relationship between kinds of ions in the layered inorganic compounds at the time of intercalation and properties of the compounds such as thermal stability, antifungal performance, etc.

\section{Experimental}

\subsection{Materials}

We used synthetic fluoride substituted mica (abbreviated to Na-MICA), whose ions between layers are $\mathrm{Na}$, as a layered inorganic ion exchanger. $\mathrm{Ca}\left(\mathrm{NO}_{3}\right)_{2} \cdot 4 \mathrm{H}_{2} \mathrm{O}$ and $\mathrm{Cu}\left(\mathrm{NO}_{3}\right)_{2} \cdot 3 \mathrm{H}_{2} \mathrm{O}$ were used for substitution of ions between layers of Na-MICA. The antifungal agent (abbreviated to TCZ), whose structure is shown in Fig.1, was used for

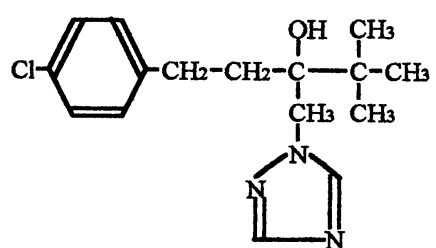

Fig. 1 Structure of antifungal agent $\alpha$-[2-(4-Chlorophenyl)ethyl]- $\alpha$-(1,1-di-methylethyl)-1H-1,2,4-triazolyl-(1)-ethanol 
intercalation.

\subsection{Replacement of the ion between the layer}

$20 \mathrm{~g}$ of $\mathrm{Na}-\mathrm{MICA}$ were supplied to 1 liter of $0.02 \mathrm{M} \mathrm{Ca}\left(\mathrm{NO}_{3}\right)_{2} \cdot 4 \mathrm{H}_{2} \mathrm{O}$ solution $\left(\mathrm{M}=\mathrm{mol} \mathrm{dm}{ }^{-3}\right)$, which was agitated at $60{ }^{\circ} \mathrm{C}$ for $24 \mathrm{~h}$. It was filtrated and washed with pure water to obtain MICA whose ions between layers are $\mathrm{Ca}^{2+}$ (abbreviated to Ca-MICA). Similarly, $20 \mathrm{~g}$ of Na-MICA were supplied to $1 \mathrm{dm}^{3}$ of $0.02 \mathrm{M}$ $\mathrm{Cu}\left(\mathrm{NO}_{3}\right)_{2} \cdot 3 \mathrm{H}_{2} \mathrm{O}$ solution, which was agitated at $60{ }^{\circ} \mathrm{C}$ for $24 \mathrm{~h}$. It was filtrated and washed with pure water to obtain MICA whose ions between layers are $\mathrm{Cu}^{2+}$ (abbreviated to $\left.\mathrm{Cu}-\mathrm{MICA}\right)$.

\subsection{Intercalation of the antifungal agent}

$4 \mathrm{~g}$ of TCZ and $16 \mathrm{~g}$ of Na-MICA were mixed well, then $20 \mathrm{~g}$ of pure water were added and mixed to form paste. After drying at $120{ }^{\circ} \mathrm{C}$ for $24 \mathrm{~h}$, intercalation was performed to obtain the compound of TCZ and Na-MICA (abbreviated Na-MICA-TCZ). The same operation was done for other kinds of ions to obtain Ca-MICA-TCZ and Cu-MICA-TCZ, respectively. Intervals between layers were measured by a powder X-ray diffraction method (shift of the minimum angle $(2 \theta)$ peak to the lower angle side) to confirm intercalation. 1 $\mathrm{g}$ of the obtained compound was dissolved in $100 \mathrm{~cm}^{3}$ of ethanol and filtered, then the amount of organic material in residue was measured by the powder X-ray diffraction method to confirm bonding types.

\subsection{Antifungal performance evaluation tests}

The minimum inhibitory concentration to fungi (abbreviated to MIC) was measured by an agar dilution method. Its procedure is as follows. Potato dextrose agar media containing different concentrations of the compound obtained by the above methods were prepared in sterilized dishes. A certain amount of fungus spore suspension was inoculated to all dishes, which were incubated at the temperature of $25{ }^{\circ} \mathrm{C}$, relative humidity of $90 \%$ or higher, for one week. In the dishes whose fungus growth was completely inhibited, the minimum concentration of the compound (MIC) was measured. Aspergillus niger IFO 6341 (abbreviated to A.niger) and Cladosporium cladosporioides IFO 6348 (abbreviated to Clado.) were used as fungi to be tested.

\subsection{Heat-resistant tests}

Heat weight change (abbreviated to TG) was measured in air flow with the temperature elevation speed of $20^{\circ} \mathrm{C} / \mathrm{min}$ to see weight reduction starting temperature.

\section{Results and Discussion}

\subsection{Bonding types of compounds}

Powder X-ray diffraction patterns of Na-MICA, Na-MICA-TCZ, and Na-MICA-TCZ after ethanol washing are shown in Fig. 2. Patterns of compounds with Ca-MICA are shown in Fig. 3, and patterns of compounds with Cu-MICA are shown in Fig. 4. Layer intervals of all compounds spread, which demonstrate the intercalation of TCZ.

Table 1 Amounts of organic material of each compound with no washing and with washing by ethanol

\begin{tabular}{ccc}
\hline Compound & No washing & $\begin{array}{c}\text { Washed by } \\
\text { ethanol }\end{array}$ \\
\hline Na-MICA-TCZ & $19.5($ wt\%) & $1.1($ wt\%) \\
Ca-MICA-TCZ & $19.6($ wt\%) & $1.5($ wt\%) \\
Cu-MICA-TCZ & $19.8($ wt\%) & $19.5($ wt\%) \\
\hline
\end{tabular}
Amounts of organic material of each compound with no washing and with washing by ethanol are shown in Table 1 . When ions between layers were $\mathrm{Na}^{+}$and $\mathrm{Ca}^{2+}$, most organic material did not remain after the washing, and layer intervals returned to original sizes. 
These results indicate that all antifungal agents were liquated out. They also indicate that the bonding power of $\mathrm{Na}, \mathrm{Ca}-\mathrm{MICA}$ and TCZ is very weak.

On the other hand, when ions between layers are $\mathrm{Cu}^{2+}$, the amount of organic material did not decrease after washing by ethanol, and layer intervals did not change. It is considered that the bonding power of $\mathrm{Cu}-\mathrm{MICA}$ and TCZ is strong. It is known that layered inorganic ion exchangers such as montmorillonite can intercalate molecules other than ions, such as water molecules and organic polar molecules, between their layers. Bonds between Na, Ca-MICA and TCZ are considered to belong to this type (intermolecular bonding).[3] On the other hand, metals, such as $\mathrm{Cu}$, tend to form complex compounds with organic polarity molecules. Since TCZ becomes Cu-TCZ complex ions between the layers, it is thought that the bond with MICA is an ionic bond.

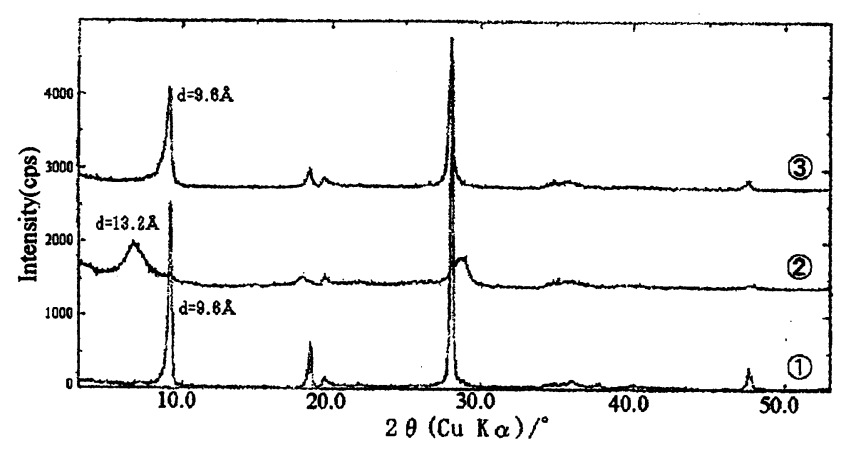

Fig. $2 \mathrm{X}$-ray diffraction patterns

(1): Na-MICA, (2): Na-MICA-TCZ,

(3): Na-MICA-TCZ washed by ethanol

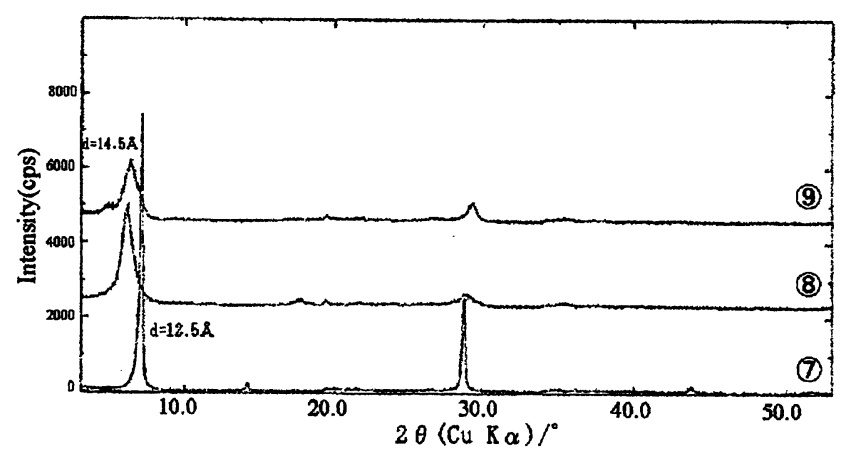

Fig. $4 \mathrm{X}$-ray diffraction patterns

(7): Cu-MICA, (8): Cu-MICA-TCZ,

(9): Cu-MICA-TCZ washed by ethanol

\subsection{Antifungal performance}

The MIC measurement results of all compounds are shown in Table 2. While MIC values of Na-MICA-TCZ and Ca-MICA-TCZ are almost at the same level, MIC of Cu-MICA-TCZ was larger than those of others. This means that Cu-MICA-TCZ has weak antifungal effect. It is considered that the difference in the antifungal

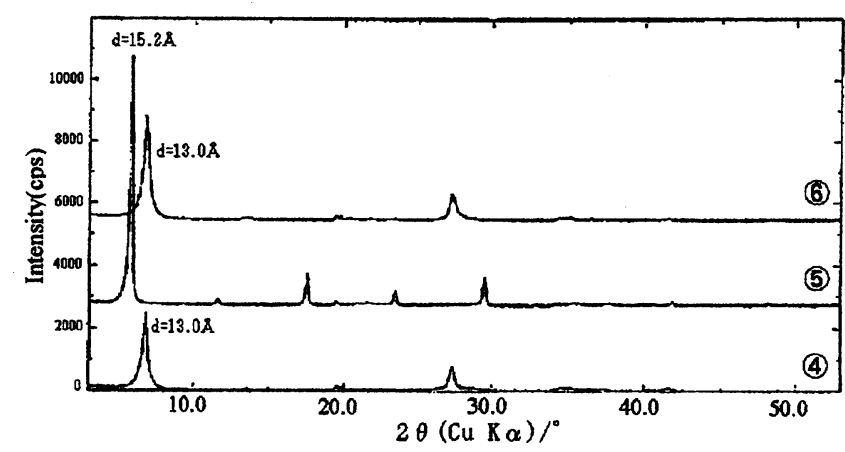

Fig. $3 \mathrm{X}$-ray diffraction patterns

(4): Ca-MICA, (5): Ca-MICA-TCZ,

(6): Ca-MICA-TCZ washed by ethanol
Table 2 Antifungal performance of compounds

\begin{tabular}{ccc}
\hline \multirow{2}{*}{ Compounds } & \multicolumn{2}{c}{ MIC $/ \mathrm{mg} \mathrm{dm}^{-3}$} \\
\cline { 2 - 3 } & A. niger & Clado. \\
\hline Na-MICA-TCZ & 200 & 100 \\
Ca-MICA-TCZ & 100 & 100 \\
Cu-MICA-TCZ & 400 & 200 \\
\hline
\end{tabular}


effect is based on the difference in the bonding type of the antifungal agent and MICA. That is, since the bonds between Na, Ca-MICA and TCZ are weak intermolecular bonding, antifungal agents are easily released, so the antifungal effect becomes high. Since Cu-MICA and TCZ are ionic bonds, the antifungal agents cannot be easily released, so the antifungal effect becomes low.

\subsection{Heat resistance}

TG curves of all compounds are shown in Fig. 5. In the case of TCZ alone, the loss in quantity starts at around $190{ }^{\circ} \mathrm{C}$. But in the case of Ca-MICA-TCZ, the loss in quantity starts around $290{ }^{\circ} \mathrm{C}$, which means that its heat resistance is improved significantly. In the cases of Na-MICA-TCZ and Cu-MICA-TCZ, the loss in quantity starts around $200{ }^{\circ} \mathrm{C}$. This means that the heat resistance is not improved.

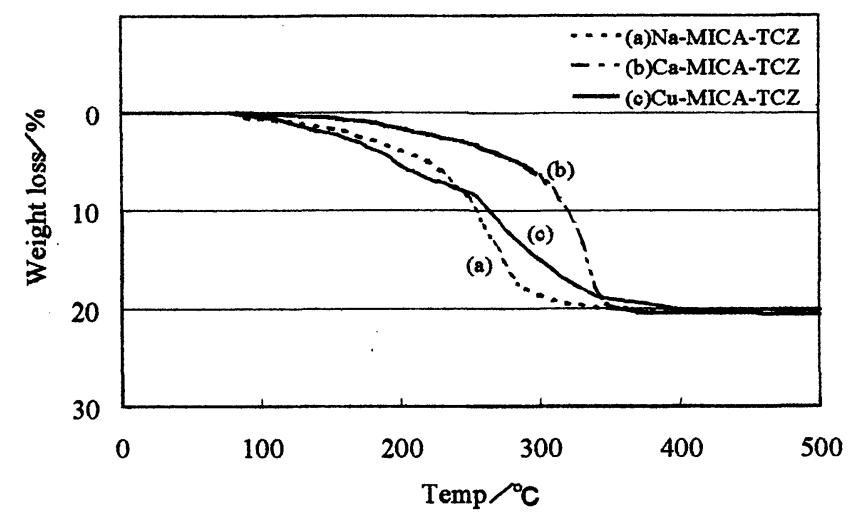

Fig. 5 TG curves of (a)Na-MICA-TCZ, (b)Ca-MICA-TCZ, and (c)Cu-MICA-TCZ

X-ray diffraction patterns of the compounds show that heat-resistant Ca-MICA-TCZ has a narrower and higher peak, which is layer intervals (see Fig. 3). It is considered that its firm layer structure realized its high heat resistance. Conversely, the peaks of Na-MICA-TCZ and Cu-MICA-TCZ are wide and low (see Fig. 2 and 4). It means that their layer structures are disordered and have portions where antifungal agents are exposed. It can be a reason for low heat resistance. In the case of intermolecular bonding type compound, whether the layer structures of MICA intercalating antifungal agents is maintained or not seems to depend on hydration energy of ions in layers. Since hydration energy of $\mathrm{Na}$ is small, Na-MICA takes in water in its layers and swells infinitely.[4] It can return to the original layer structure after drying, but if it has TCZ, it will be inhibited from returning to the perfect layer structure.

On the other hand, as hydration energy of $\mathrm{Ca}$ is large, Ca-MICA attracts layers even if it in in the state of hydration, so its layer structure is maintained though its layer intervals can reach to $1.8 \mathrm{~nm}$ at their maximum.[4] Therefore, TCZ is intercalated into layers whose structure is maintained, so it is considered that the layer structure of the compound is also maintained.

\section{References}

1) S. Yamanaka, M. Hattori, SURFACE, 19, 54-65 (1981).

2) H. Hayama, The Chemical Society of Japan, 11,806-810 (1997).

3) A. Oya, Gypsum \& Lime, 239, 267-274 (1992).

4) T. Watanabe, Smectite study group report, 1, 2-15 (1991). 